\title{
Correction for Camera Roll in a Perspectively Distorted Image: Cases for 2 and 3 point perspectives
}

\author{
Avinash N. ${ }^{*}$ and Murali S. \\ * Department of I.S.E., P.E.T Research Center, P.E.S. College of Engineering,Mandya 571401,INDIA \\ wittybot@gmail.com, ${ }^{+}$nymurali@yahoo.com
}

Received 8 May 2008; accepted 8 July 2009

\begin{abstract}
We propose a method for correction of camera roll in a perspectively distorted image. Here we employ the rectification of the horizon line formed from the vanishing points in order to bring back the image rotation caused from the camera roll. In teleconference systems, surveillance system, aerial images of buildings, mobile robots visual system etc., the range of camera's view spreads with pan, tilt and roll. It is intended to take omni-directional images for wide range coverage, but this invariably introduces rotation in many images due to the roll of the camera. This paper presents the method for camera roll correction by using the perspective distortion in images captured from a pan/tilt/roll camera control system. The proposed method works on two cases, viz. Calibrated and uncalibrated cameras. Calibrated camera with intrinsic calibration of the camera such as optical center and the focal length of the camera in cases of two point perspectives is considered and in case of three point perspective uncalibrated camera is considered and the camera is partially calibrated for the camera principal point and the roll is performed subsequently. The result comparison made reveals more about the usability of the algorithm in case of calibrated and uncalibrated cases of the camera used.
\end{abstract}

Keywords: Pan Tilt Roll of Camera, Optical Center of Camera, Vanishing Points, Horizon Line, principal point (center of focus).

\section{Introduction}

This paper focuses mainly on the correction of camera roll to rectify the image in order to bring the inclined objects as seen in the image to a necessary vertical position. While capturing pictures or videos in a camera, the device most of the time is hand held or fixed on an irregular surface. This invariably brings about the tilt and roll effect in the image captured. In this regard we propose an algorithm to a camera system which can automatically correct the tilt and roll

Correspondence to: Avinash N. <wittybot@gmail.com>

Recommended for acceptance by Umapada Pal and P. Nagabhushan

ELCVIA ISSN: 1577-5097

Published by Computer Vision Center / Universitat Autonoma de Barcelona, Barcelona, Spain 
effect caused by the irregular positioning of the camera as the image is captured. In this paper we propose a novel method for roll rectification for a camera system based on the vanishing points situation.

The object in the space is represented by some points $\left(\mathrm{x}_{\mathrm{i}}, \mathrm{y}_{\mathrm{i}}, \mathrm{z}_{\mathrm{i}}\right)$ with respect to the camera co-ordinate system shown in figure 1. Any change of the camera orientation is about these axes. Different changes about these axes are termed by different terms, namely pan, tilt and roll.

- Pan is the change in camera orientation about y axis. That is the rotation of the camera about the y axis.

- Tilt is the change in camera orientation about the $\mathrm{x}$ axis. That is the rotation of the camera about the $\mathrm{x}$ axis.

- Roll is the change in camera orientation about the $\mathrm{z}$ axis. That is the rotation of the camera about the $\mathrm{z}$ axis.

Objects such as building scenes usually yield two vanishing points when the image is captured from a long range. To give a better clarity of the objects in the image and better field of view to the camera, the image should be captured on a shorter range. In order to cover the entire object in the image, a tilt in the camera needs to be introduced and hence the third vanishing point comes in place in its image. The correction of such type of images is discussed in section 5 . More often than not, mechanical imperfections are encountered in fixing up the camera in a perfect horizontal position to capture the image. Slight variation or imperfectness in camera mounting system may introduce a roll in capturing the scene onto the image. The correction of such type of images is the main focus of this paper and is discussed in the section 4 and 5 of this paper. We have discussed two cases in correction of camera roll. viz., i. parameters of the camera known (in section 4), ii. Parameters of the camera not known (in section 5).

\section{Orientation of the Object in the Image Due to the Effect of Camera Roll}

From the properties of the camera [8], due to the type of lens used, the images acquired are always perspectively distorted. The panning of camera makes the object drift out from the field of view. This can only be used to capture dynamic scenes. Hence correction of panning is of less importance in our experimentation. Tilt in the camera introduces the third vanishing point in the image [7]. In cases of uncalibrated cameras, we use the third vanishing point based method to correct the camera roll. In the current work we are considering images which are having two or three vanishing points only. Two vanishing points cases for calibrated camera and three vanishing points cases for uncalibrated camera.

From the principles of optics [1][2], the image in the pinhole camera is created on the basis of the rectilinear propagation of light. The idea behind using the pin hole in a camera is that, the light from infinity passes through the pin hole and falls on the screen causing a perspective distortion [4][5]. The present day cameras works on the principle of a pin hole in the camera with a convex lens or a group of convex lenses. This property of light passing through the lens straight without converging is only at the optical center of the lens, like that of a pin-hole in a pinhole camera.

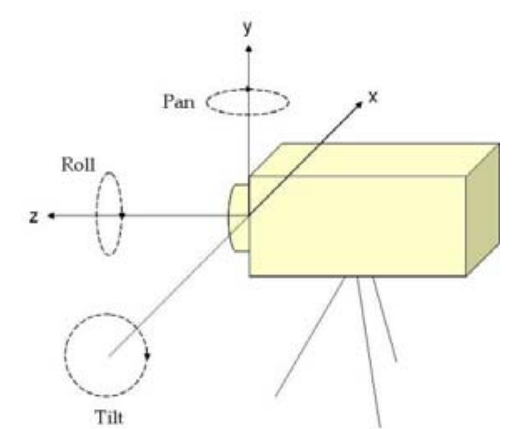

Figure 1: Combining all the views in a 3D representation of the same to show the Pan Tilt and Roll of a camera.

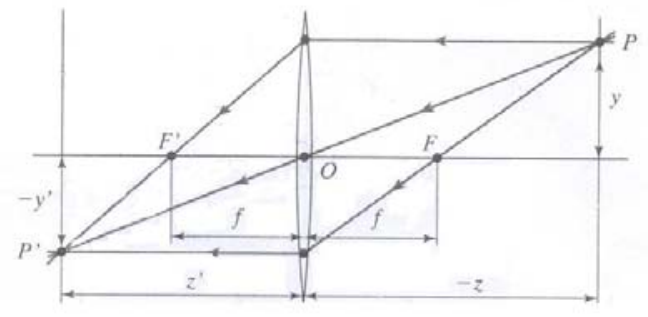

Figure 2: Ray optics of a photographic lens. 
From observation in figure 2 we can note, a point ' $\mathrm{P}$ ' located at depth ' $\mathrm{z}$ ' off the optical axis and denoted by 'PO', the ray passing through this point at the optical center of the lens ' $O$ '. It shows from Snell's law and paraxial refraction equations [1][3][6], that the ray ' $\mathrm{PO}$ ' is not refracted and that all other rays passing through ' $\mathrm{P}$ ' are focused by the lens on the point $\mathrm{P}^{1}$ with depth $\mathrm{z}^{1}$ along 'PO' such that

$$
\frac{1}{z^{1}}-\frac{1}{z}=\frac{1}{f^{1}}
$$

Where,

$$
f=\frac{R}{2(n-1)}
$$

Is the focal length of the lens.

From the equations, it can be noted that the positions of ' $\mathrm{P}$ ' and $\mathrm{P}^{1}$ are exactly the same as under pinhole perspective projection if we take $\mathrm{z}^{1}=\mathrm{f}^{1}$, since $\mathrm{P}$ and $\mathrm{P}^{1}$ lie on a ray passing through the center of the lens, but the points located at a distance ' $-\mathrm{z}$ ' from ' $\mathrm{O}$ ' are only in sharp focus when the image plane is located at a distance $\mathrm{z}$ ' from ' $\mathrm{O}$ ' on the other side of the lens that satisfies equation 1 . Letting $Z \rightarrow-\infty$ shows that ' $\mathrm{f}$ ' is the distance between the center of the lens and the plane where objects such as stars, which are effectively located at $Z \rightarrow-\infty$, focus. The two points ' $\mathrm{F}$ ' and ' $\mathrm{F}$ ', located at distance ' $\mathrm{f}$ ' from the lens center on the optical axis are called the focal points of the lens.

In practice, objects within some range of distances called depth of field or depth of focus are in acceptable focus. The field of view of the camera is the portion of scene space that actually projects onto the retina of the camera. It is pivoted about this optical center of the lens. Hence along the principal axis of the camera, which makes the shortest distance of $\mathrm{OP}^{1}$ in a camera, the image is pivoted and rotated if the camera roll occurs. Hence any correction in the camera roll to be made is about this axis of vision or the principal axis which impinges the image at center of focus the rotation has to be made to correct the image.

To overcome the camera roll, we hereby propose the following methods in the sections to follow to correct them automatically. The method we employ for the camera roll correction involves the following steps, viz.

1. Vanishing point determination

2. Determination of slope of the horizon line

3. Bringing camera center on image center

4. Image rotation.

We demonstrate the method with the geometrical framework established at each level, in the section to follow.

\section{Geometrical framework}

\subsection{Framework for Vanishing point determination-}

A vanishing point can be a finite (real) point or an infinite (ideal) point on the image or the picture plane. The vanishing point determination adopted in this paper is based on the line intersection accumulated and calculated in the Hough space, by inverse Hough transformation approach. For all vanishing point determination techniques in the spatial 
domain, the first step involved to determine the vanishing points is the detection of the edge lines that contribute for the vanishing point. Some or many of the lines detected in the image may not contribute for the determination of the vanishing points. Further all the combinations of the detected lines are used for the determination of individual points which might contribute for the vanishing point. But due to the camera sensor errors in acquiring the image, and the motion errors the edges of the objects which make the lines in the image do not contribute to intersect at the same point always. But instead, they meet nearby the actual vanishing point. Thus a large number of points are generated upon intersection of all these lines, which are all within the vicinity of the actual vanishing point. Thus with the large set of points collected, averaging techniques are applied to approximate the existence of the vanishing point among the clustered set of points. This method of vanishing point determination is explained in this section.

\subsubsection{Line Determination-}

Lines are edges of the objects and environment present in an image. These lines may or may not contribute to form the actual vanishing point. Canny edge detector [23] is used to extract edges in the image. The locus of the point 'P' (in figure 3) which makes a trajectory along a set of points (pixels in an image which contribute to form a line L), in the spatial domain is represented by the equation 3 has a particular slope ' $m$ ' and its ' $y$ ' intercept ' $c$ '. The extent of the line varies up to the existence of points contributing for the ' $x$ ' and ' $y$ ' co-ordinates. The same line in a polar co-ordinate system takes the form mentioned in equation 4 . In this case, the range of ' $\rho$ ' and ' $\theta$ ' are fixed, and the values of ' $x$ ' and ' $y$ ' can be varied to extend the same line to any limit. This is depicted in the figure 3.

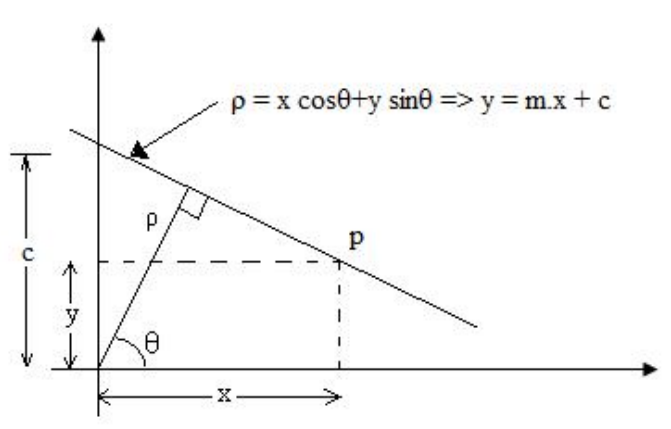

Figure 3: Equation of a line in Cartesian and polar

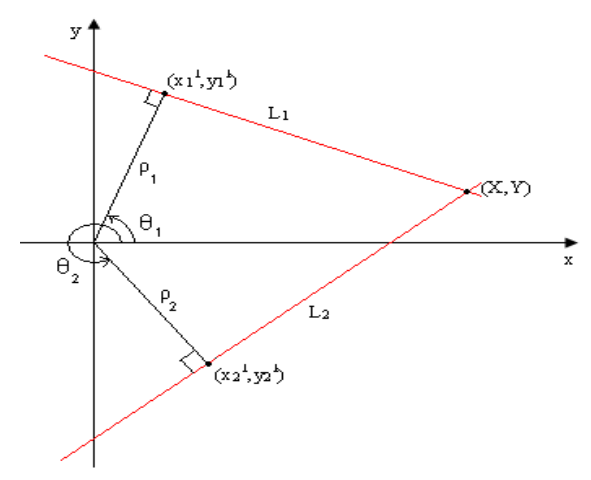

Figure 4: Representation of two lines. spaces.

$$
\begin{gathered}
y=m x+c \\
\rho=x \cdot \cos \theta+y \cdot \sin \theta
\end{gathered}
$$

For an image plane of dimension $L \times L$ pixels, the parameters ' $\rho$ ' and ' $\theta$ ' are limited. It can be readily learnt from the literature of the Hough Transformation [Hough transform] that the value of ' $\rho$ ' varies within the range [0, $\sqrt{2}$.L], while ' $\theta$ ' takes values in the range of $[-\pi / 2, \pi / 2]$. Points belonging to the same straight line in the image plane have corresponding sinusoids which intersect in a single point in the polar space. The need for calculating the number of straight lines is; there could be several straight lines in the image which intersects each other at different points in the image plane. In such case there arises a situation that more than one peak value (given by the sum of all the sinusoids of the points on the line) in the polar space is obtained. Thus by selecting the number of peak values (in descending order of their votes) equal to the number of straight lines ' $N$ ' present in the image we restrict the unwanted lines which may 
not contribute to the real vanishing point. This reduces the computational burden in vanishing point detection. Subsequently these points $((\rho, \theta)$ pairs) are selected for the inverse Hough transform based vanishing point detection.

\subsubsection{Intersection Point of any Two Lines-}

Each peak in the Hough space signifies the existence of a longer edge in the image, than any other points in the Hough space and hence a peak is formed. With this background, the intersection of multiple lines contribute to the vanishing points, as in a perspectively distorted image, all the objects appear to meet at a particular point. Hence these peak voted points of the Hough space are calculated to find the intersection between two lines to calculate the vanishing point further.

Considering lines $L_{1} \& L_{2}$ present in spatial domain as shown in figure 4 . The locus of the point $\left(\mathrm{x}_{1}, \mathrm{y}_{1}\right)$ moving along the line $\mathrm{L}_{1}$, there exists exactly one $\left(\mathrm{x}_{1}^{\prime}, \mathrm{y}_{1}^{\prime}\right)$ for the line where the center of the origin of the coordinate system to the point $\left(\mathrm{x}_{1}^{\prime}, \mathrm{y}_{1}^{\prime}\right)$ is the shortest distance along the line $\mathrm{L}_{1}$ and is termed $\rho_{1}$. The phase angle of the gradient of the line $\mathrm{L}_{1}$ is represented by $\theta_{1}$ or in other words $\theta$ is the angle measured from the abscissa to the line joining the shortest distance from origin of the coordinates to the point $\left(\mathrm{x}_{1}, \mathrm{y}_{1}\right)$.

Similarly locus of the point $\left(x_{2}, y_{2}\right)$ moving along line $L_{2}$ has a point $\left(x_{2}^{\prime}, y_{2}^{\prime}\right)$ on the line where it makes $\rho_{2}$ and $\theta_{2}$. or in other words $\left(\rho_{1}, \theta_{1}\right)$ is unique for $L_{1}$. Similarly, $\left(\rho_{2}, \theta_{2}\right)$ is unique for $L_{2}$.

If,

$$
\theta_{1} \neq \theta_{2} \Rightarrow L_{1} \nVdash L_{2}
$$

i.e. if,

$$
\theta_{1} \neq \theta_{2} \exists(X, Y) \mid(X, Y) \in L_{1} \text { and } L_{2}
$$

Therefore from equation 4 it implies,

$$
\begin{aligned}
& \rho_{1}=X \cdot \cos \theta_{1}+Y \cdot \sin \theta_{1} \\
& \rho_{2}=X \cdot \cos \theta_{2}+Y \cdot \sin \theta_{2}
\end{aligned}
$$

This is because point $(\mathrm{X}, \mathrm{Y})$ is common to both lines $\mathrm{L}_{1}$ and $\mathrm{L}_{2}$.

Thus solving for the above equations,

$$
\begin{gathered}
X=\frac{\rho_{1}-Y \cdot \sin \theta_{1}}{\cos \theta_{1}}=\frac{\rho_{2}-Y \cdot \sin \theta_{2}}{\cos \theta_{2}} \\
Y=\frac{\left(\left(\frac{\rho_{1}}{\cos \theta_{1}}\right)-\left(\frac{\rho_{2}}{\cos \theta_{2}}\right)\right)}{\tan \theta_{1}-\tan \theta_{2}}
\end{gathered}
$$

Thus from equations 7 and 8 , it can be inferred that given two pairs of $(\rho, \theta)$ values (from the Hough plot), it is possible to find the intersection point in the image plane of the corresponding lines which represent the lines correspondingly by calculation directly on the points in Hough space.

Applying for all combinations of lines selected by the $(\rho, \theta)$ peak values as in the equations 7 and 8 and selecting two at a time, correspondingly one $(\mathrm{X}, \mathrm{Y})$ pair is obtained. The number of pairs of $\mathrm{X}$ and $\mathrm{Y}$ values obtained from the two $(\rho, \theta)$ values selected 2 at a time, for all combinations, is given by the relation. 


$$
{ }^{N} C_{2}=\frac{N !}{(N-2) ! \times 2 !}=K
$$

Where $\mathrm{N}$ is the number of $(\rho, \theta)$ pairs selected and also equal to the number of lines in the image ' $\mathrm{N}$ '. The value of ${ }^{\mathrm{N}} \mathrm{C}_{2}$ is the number of $(\mathrm{X}, \mathrm{Y})$ pairs that are calculated using the equations 7 and 8 where in $\mathrm{X}$ and $\mathrm{Y}$ are computed individually. Thus a large number of points are generated upon intersection of all these lines, which are all within the vicinity of the actual vanishing point. Thus it is required to find a center among these points. For this section that follows explains.

\subsubsection{Clustering of Scattered Points-}

From the equation 9, ' $\mathrm{K}$ ' number of points is obtained by the intersection of all possible combination of lines. The intersection points obtained are scattered all around the space due to the existence of multiple vanishing points and hence these are further need to be clustered to bring about the group of possible vanishing point all to one side together. Before clustering is made, a threshold of the distance should be fixed based on a heuristic knowledge. Most of the times, we fix up a threshold of a distance of $50 \%$ of the diagonal distance of the image on hand. This is because; most of the times the images we consider have only one, two or a maximum of 3 vanishing points and the scatter pattern of these points are differentiable into different patterns. From the geometry of the focus of the object captured, is usually perpendicular mutually to each other. This means, the vanishing points we get from these faces of the objects are distinctly away from each other and almost at the extreme corners of the image or even outside the image.

We use single linkage clustering algorithm [25] to fix up the different clustered points from the K number of points obtained. Subsequently median of the points is applied in each cluster individually to estimate the vanishing points in the image. This is because, the median gives the existence of the actual central value among the cluster of points. Whereas the other measures of central tendencies [24] like mean and mode are the averaged point among all the points in the cluster. The chances of a point lying at a larger distance than that of the frequently found ones, then the error propagates into the calculation of mean. Hence, median is preferred over mean in estimating the vanishing point among the clustered set of points.

Algorithm of the method employed to determine vanishing points is as below.

Step 1. Read the input image and normalize the same.

Step 2. Canny edge detector is applied to the normalized image which yields a binary image with only the edge information.

Step 3. The image is then subjected to Hough Transform. Number of straight lines present in the created image is stored globally as $\mathrm{N}$.

Step 4. Select only the maximum votes of first $\mathrm{N}$ number of cells in the Hough space for further calculation.

Step 5. Arrange these votes in descending order of their values. Select only the first $\mathrm{N}$ number of votes among these set of points in the Hough space. Store these in a data structure.

Step 6. Calculate the $X \& Y$ as derived in the section 3.1.2 for all pairs of $(\rho, \theta)$ pairs.

Step 7. Carry out step 6 for all $\mathrm{N}$ number of Hough points, selecting 2 at a time. Thus performing ${ }^{\mathrm{N}} \mathrm{C}_{2}$ combinations of points, to get their corresponding intersection points i.e. the $(X, Y)$ values into a data structure. These are the tentative vanishing point coordinates. 
Step 8. Considering all the co-ordinates obtained from step 7, start performing an agglomerative clustering technique (single linkage clustering in this case) up to which these points forms different clusters and would not further classify into different groups.

Step 9. Determine the vanishing points by finding the median points of $(X, Y)$ pairs in each cluster, for all the clusters formed in step 8 .

Step 10. As many clusters are formed, that many vanishing points are thus determined.

\subsubsection{Results-}

The result of vanishing point determination at each stage is shown in

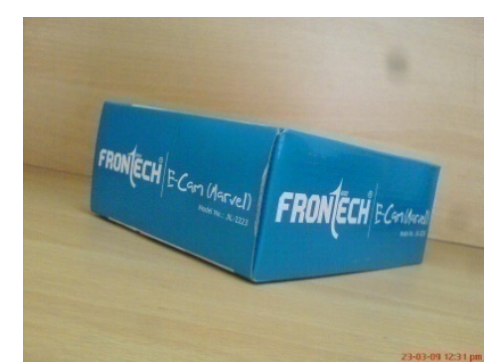

(a)

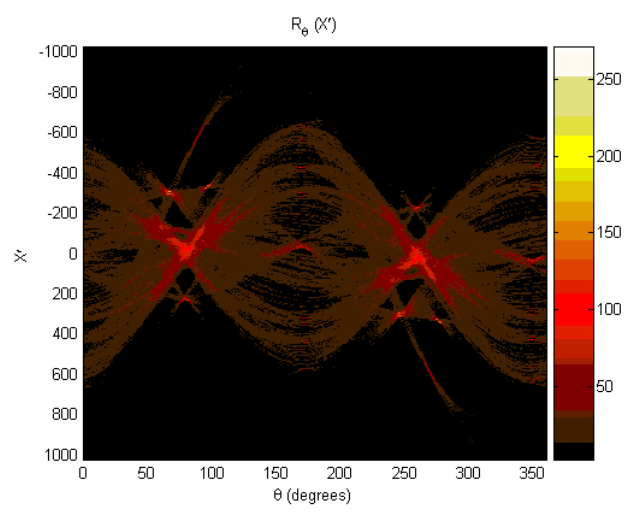

(d)

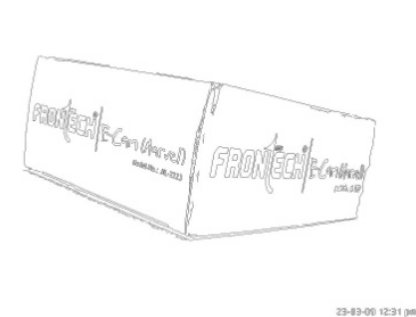

(b)

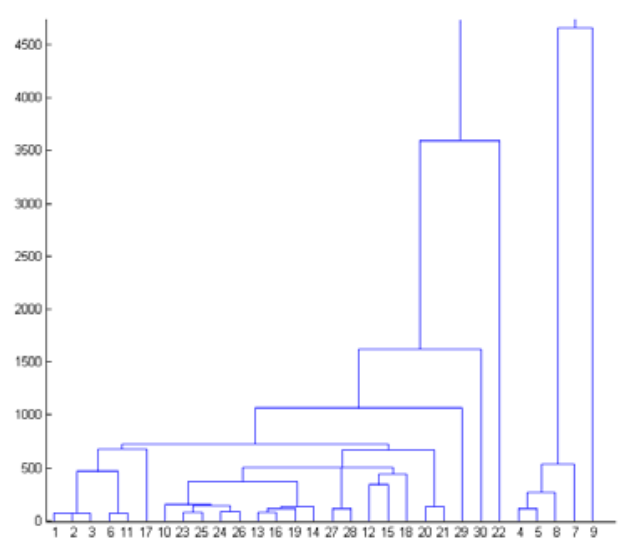

(e)

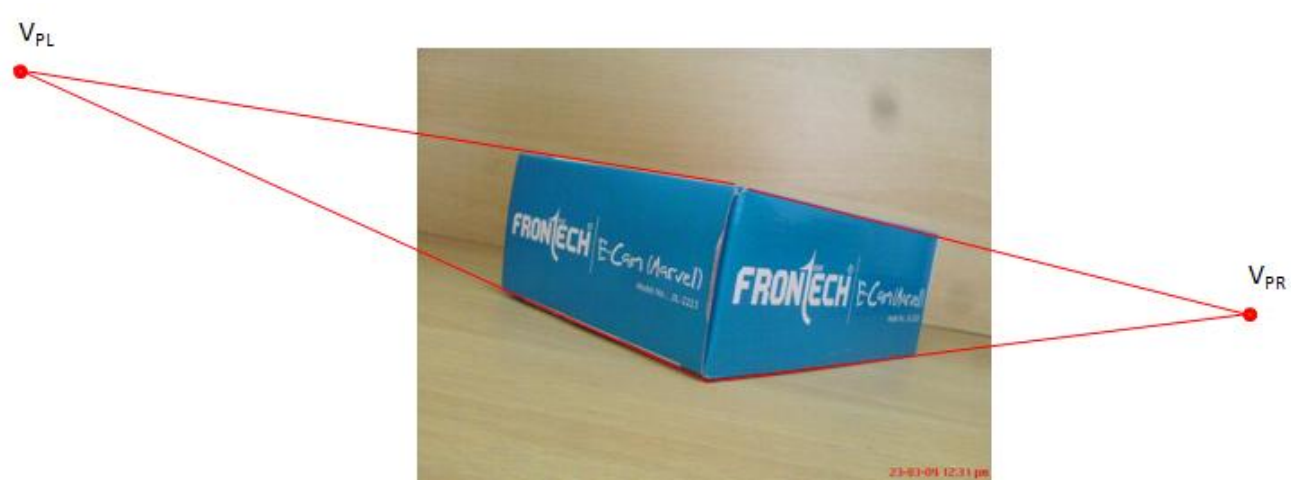

(f)

Figure 5: Process of vanishing point determination in different stages. (a) Original input image for the determination of vanishing point. (b) Canny edge detected image of the input image. (c) ' $N$ ' number of lines detected from the Canny edge detected image with the application of Hough Transform. (d) Voted space in the Hough space with peak values varying from black to white, while white with maximum count. (e) Application of clustering for the intersected points of the non parallel lines, by picking peak values from the Hough space and calculating the intersection points using equations 7 and 8 . (f) Two vanishing points determined are shown. 


\subsection{Slope determination of the Horizon Line}

The vanishing point is determined from the Inverse Hough Transformation based method [21]. The vanishing points found out are termed as $\mathrm{V}_{\mathrm{PL}}=\left(\mathrm{x}_{1}, \mathrm{y}_{1}\right)$ and $\mathrm{V}_{\mathrm{PR}}=\left(\mathrm{x}_{2}, \mathrm{y}_{2}\right)$.

The slope of the line joining these two points (Horizon Line [14]) is given by the relation,

$$
m=\frac{\left(y_{2}-y_{1}\right)}{\left(x_{2}-x_{1}\right)}
$$

The angular orientation of this horizon line is

$$
\varphi=\tan ^{-1} m
$$

\subsection{Making camera center as Image center}

In order to be able to avoid parallax shift in the images it is necessary to pivot the image about the camera's center of perspective, which turns out to be the center of the entrance pupil of the lens[19][20].

To bring the difference of the pixel co-ordinates of the image center and the optical center in the image, we add up zero values to the border of image as shown in figure 7(c). Figure 7(b). shows the actual image, where in a object is exactly vertical to the ground but appears to be tilted due to the effect of the roll introduced in the camera. By preparing the image with some zero values at the borders (as shown in figure 7(c)) and bringing the co-ordinates of the image center and the optical center to a common point, we perform the estimation of vanishing point. After finding the vanishing points, the points are joined to draw a straight line. This is the Horizon Line [14]. We estimate the slope of the horizon line and there on find out at what degree of inclination the camera is rolled from equation 11.

\subsection{Image Rotation}

A rotation matrix is a matrix which when multiplied by a vector has the effect of changing the direction of the vector but not its magnitude. Since the rotation of the image is done along the image's horizontal and vertical axes, the following relation holds good for the performing of image rotation about the principal point as the principal point is now brought on to the image center by the application of method in section 3.3.

$$
R=\left[\begin{array}{cc}
\cos \theta & \sin \theta \\
-\sin \theta & \cos \theta
\end{array}\right]
$$

The consideration of square pixels brings about noise and pixel distortion along with some distortions in the rotated matrix, due to the multiplication of matrices. This can be overcome by pixel approximation by nearest neighbourhood method. 


\subsection{Finding the orthocenter of a triangle}

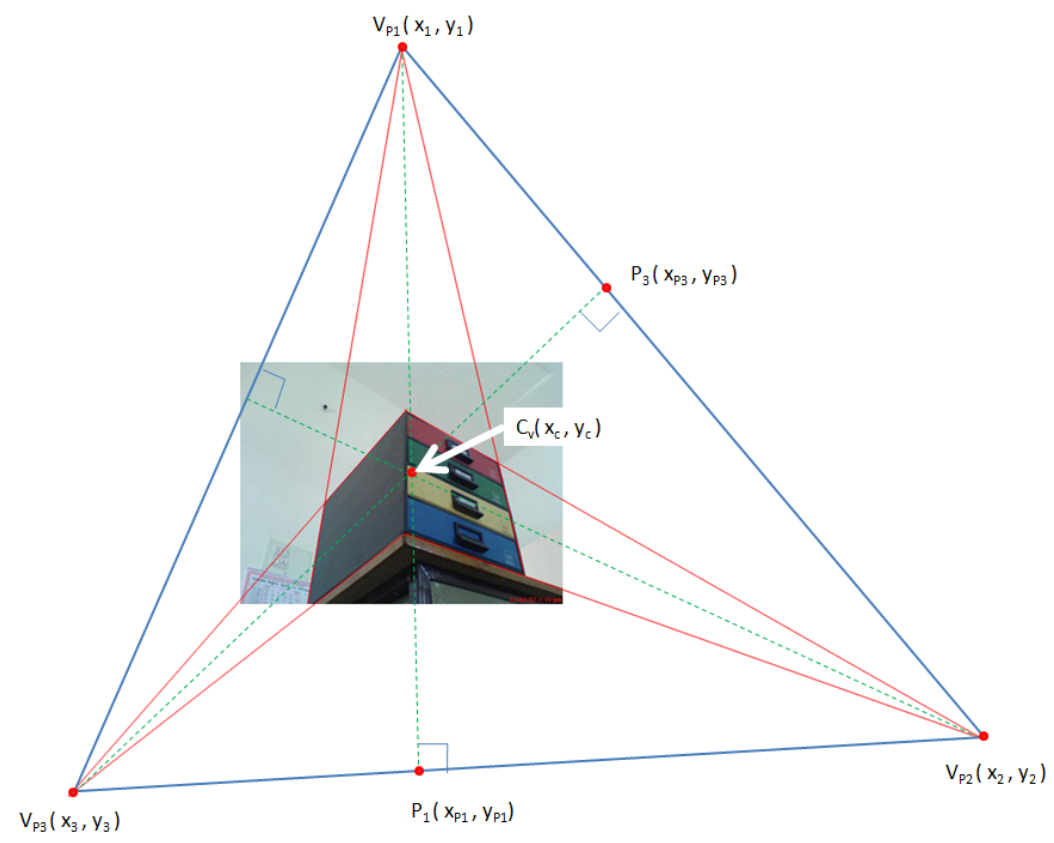

Figure 6: Orthocenter of the triangle formed from 3 vanishing points given by $C_{v}\left(x_{c}, y_{c}\right)$.

The orthogonal projection of $\mathrm{V}_{\mathrm{P} 1}$ onto the line $\overrightarrow{V_{P 2} V_{P 3}}$ is the point $\mathrm{P}_{1}(\mathrm{xP} 1, \mathrm{yP} 1)$. Similarly $\mathrm{V}_{\mathrm{P} 3}$ is orthogonally projected onto $\overrightarrow{V_{P 1} V_{P 2}}$ to get $\mathrm{P}_{3}\left(\mathrm{x}_{\mathrm{P} 3}, \mathrm{y}_{\mathrm{P} 3}\right)$.

The co-ordinates of $P_{1}$ is given by

$$
P_{1}\left(x_{P 3}, y_{P 3}\right)=\left(x_{2}+r_{1} . l_{1}, y_{2}+r_{1} . m_{1}\right)
$$

Where $\left(\mathrm{l}_{1}, \mathrm{~m}_{1}\right)$ are the directional cosines of $\overrightarrow{V_{P 2} V_{P 3}}$ and is given by

$$
\left(l_{1}, m_{1}\right)=\left(\frac{a_{1}}{\sqrt{a_{1}^{2}+b_{1}^{2}}}, \frac{b_{1}}{\sqrt{a_{1}^{2}+b_{1}^{2}}}\right)
$$

And ' $\mathrm{r}$ ' is the radius vector, given by

$$
r_{1}=\frac{a_{1}\left(x_{1}-x_{2}\right)+b_{1}\left(y_{1}-y_{2}\right)}{a_{1} \cdot l_{1}+b_{1} \cdot m_{1}}
$$

Where $\left(\mathrm{a}_{1}, \mathrm{~b}_{1}\right)$ are the directional ratios of $\overrightarrow{V_{P 2} V_{P 3}}$, given by

$$
\left(a_{1}, b_{1}\right)=\left[\left(x_{3}-x_{2}\right),\left(y_{3}-y_{2}\right)\right]
$$

Thus with the given co-ordinates, the equation of line joining points $\mathrm{V}_{\mathrm{Pl}}\left(\mathrm{x}_{1}, \mathrm{y}_{1}\right)$ and $\mathrm{P}_{1}\left(\mathrm{x}_{\mathrm{P} 1}, \mathrm{y}_{\mathrm{P} 1}\right)$ is given by

$$
\left(y-y_{1}\right)=m_{P 1} \cdot\left(x-x_{1}\right)
$$

Where the slope of the line represented by $\mathrm{m}_{\mathrm{P} 1}$ is given by

$$
m_{P 1}=\frac{\left(y_{P 1}-y_{1}\right)}{\left(x_{P 1}-x_{1}\right)}
$$

Similarly equation of the line joining points $\mathrm{V}_{\mathrm{P} 3}\left(\mathrm{x}_{3}, \mathrm{y}_{3}\right)$ and $\mathrm{P}_{3}\left(\mathrm{x}_{\mathrm{P} 3}, \mathrm{y}_{\mathrm{P} 3}\right)$ is given by

$$
\left(y-y_{3}\right)=m_{P 3} \cdot\left(x-x_{3}\right)
$$

Where the slope of the line represented by $\mathrm{m}_{\mathrm{P} 3}$ is given by

$$
m_{P 3}=\frac{\left(y_{P 3}-y_{3}\right)}{\left(x_{P 3}-x_{3}\right)}
$$


For non parallel lines in equation 17 and 19 , they share a common point $\mathrm{C}_{\mathrm{v}}\left(\mathrm{x}_{\mathrm{c}}, \mathrm{y}_{\mathrm{c}}\right)$ at their intersection. Thus at $\left(\mathrm{x}_{\mathrm{c}}, \mathrm{y}_{\mathrm{c}}\right)$ it satisfies both the equation and thus they take the form

$$
\begin{aligned}
& x_{c}=\left(\frac{y_{c}-y_{1}}{m_{P 1}}\right)+x_{1} \\
& x_{c}=\left(\frac{y_{c}-y_{3}}{m_{P 3}}\right)+x_{3}
\end{aligned}
$$

Solving for equations 21 and 22, we get

$$
\begin{gathered}
x_{c}=\frac{m_{P 1} \cdot x_{1}-m_{P 3} \cdot x_{3}-y_{1}+y_{3}}{m_{P 1}-m_{P 3}} \\
y_{c}=m_{P 1} \cdot x_{c}-m_{P 1} \cdot x_{1}+y_{1}
\end{gathered}
$$

\section{Roll Rectification from known camera parameters}

In the figures 7(a) and (b), we have considered images of resolution of 640x480 pixels. The actual center of the image is supposed to be at co-ordinate $(320,240)$. But as per the camera calibration results obtained by the algorithm in [13], it is found that the optical center of the camera is supposed to be found at the co-ordinate $C_{v}=(337,227)$. This difference in shift of the optical center and the image center is due to the fabrication errors while manufacturing the lenses to the camera. Optical center is the actual center of focus in the scene upon which the image is pivoted. This is also called as principal point of focus [14]. Hence, rotation made to the image is supposed to be performed about this point in the image.

This is because the horizon line is supposed to be parallel to the horizontal axis of the image, as explained in section 2. Rotate back the image by the angle of inclination of the horizon line about the new image center. Crop the image if needed for retaining only the necessary information. This method gives a roll rectified image (as shown in figure 7(d)). The step wise process is shown in figures 7 (b) through (d).

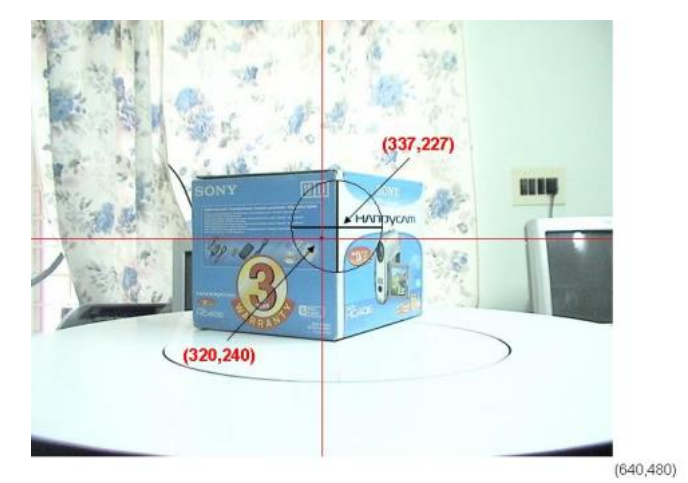

Figure 7 (a): Representation of the image center and the optical center in the image. Image dimension being (640,480), the image center is at $(320,240)$ and the optical center is at $(337,227)$.

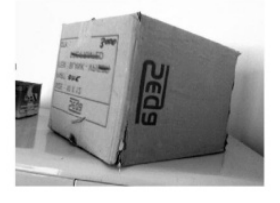

Figure 7 (b): A vertical object captured at some unknown roll of the camera.

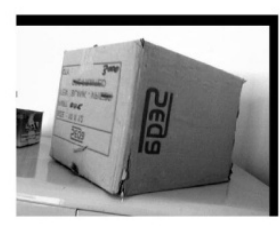

Figure 9 (c): Padding up of zero values to the original image to bring the image center upon optical center.

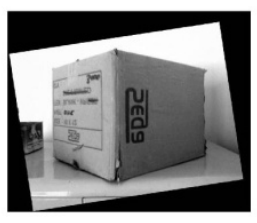

Figure 7 (d): Rotate the image about the new image center with the calculated degree of inclination of the horizon line.

Figure 7: Stages in image rotation 
Figure 7(b) shows the image acquired from the same camera. In order to make the center of focus as the center of image, we add up strips of zero values above and to the right of the image equal to the difference in the ordinate and abscissa values of the two centers of our consideration. This image of the padded zero values is depicted in figure 7(c). The image rotation about the angle equal to the slope of the horizon is applied to figure 7(c) to obtain the figure 7(d) in order to bring about the correction of camera roll, as the given equation 12 brings about rotation about the center of the image. The other results of images acquired are shown in table 1.

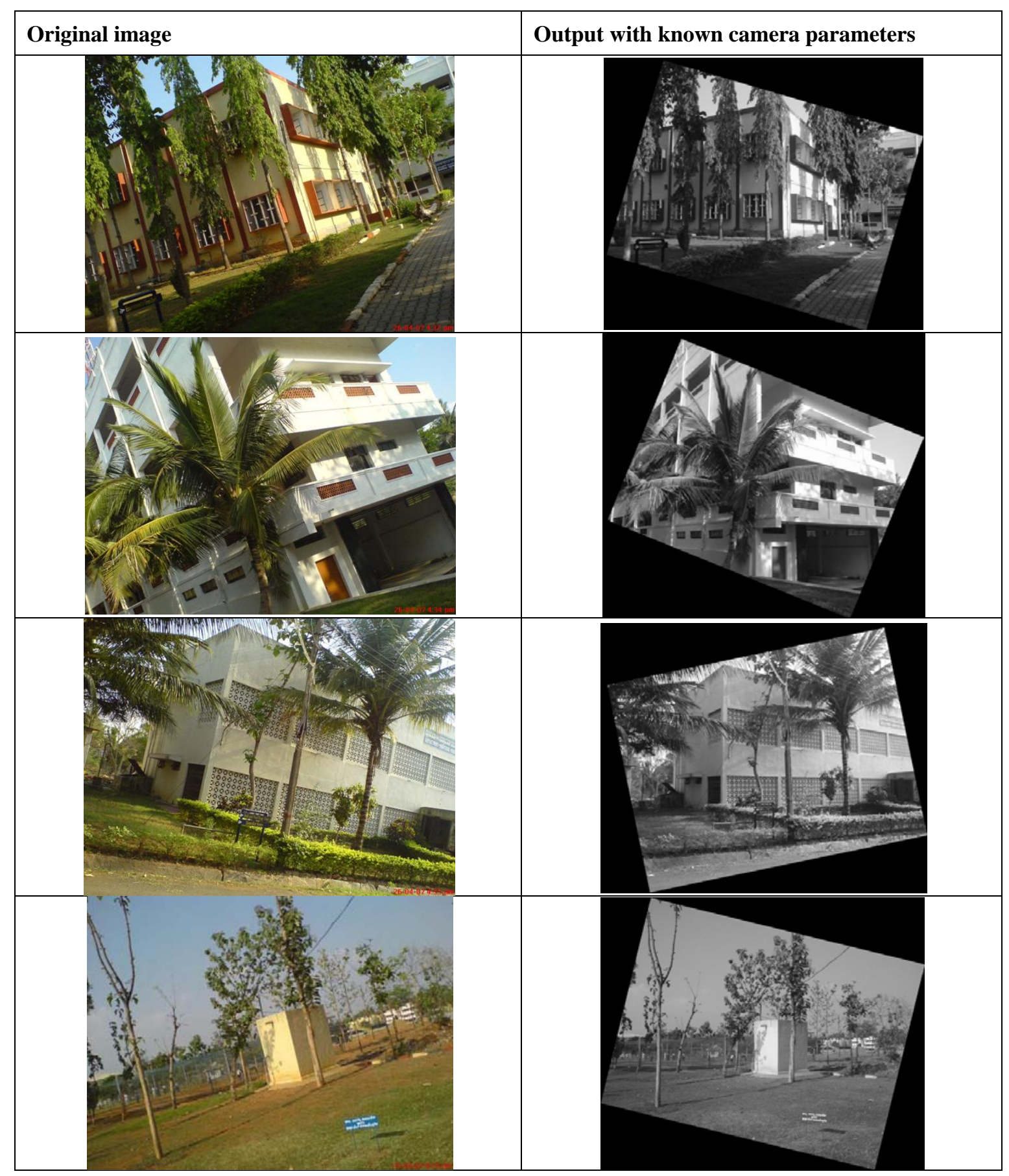

Table 1: Roll rectification input and output images for calibrated cameras. 


\section{Algorithm:}

Step 1: Load the given image.

Step 2: Find out the vanishing points in the loaded image.

Step 3: Calculate the slope of the line joining the two vanishing points as in equation 10.

Step 4: Find the degree of inclination of the image by applying the arctangent (equation 11) to the slope and name it $\varphi$.

Step 5: About the optical center of the camera which is depicted on the image by the co-ordinates $C_{v}=\left(x_{c}, y_{c}\right)$, rotate the image by an angle equal to $-\varphi$.

Step 6: To accomplish the above step, follow the steps 'a' through ' $e$ '

a) Check the image center and the optical center in the image.

b) If they are the same points, then skip step 'c' else follow step 'c'

c) Add padding zero values to the borders of the image so as to bring the optical center equal to the image center.

d) Rotate the image using the image rotation matrix, about the image center, with the above specified angle.

Step 7: Stop

\section{Roll Rectification from unknown camera parameters}

\section{(Roll Rectification from Images Having 3 Vanishing Points:)}

As mentioned in section 2, it is learnt that the camera roll is brought about the center of focus and the axis of vision is the pivot point. In cases of calibrated camera, the image rectification can be brought about the center of focus of the image. But in case of uncalibrated camera it is required to partially calibrate the camera and find out the center of focus of the image even before the roll is actually performed on the image.

From the literature [22], it is found that the orthocenter of the image is the center of focus (as mentioned as principal point in [22] in chapter 8). From figure 6, the vertices VP1, VP2, VP3 with their corresponding co-ordinates being $(\mathrm{x} 1, \mathrm{y} 1),(\mathrm{x} 2, \mathrm{y} 2)$ and $(\mathrm{x} 3, \mathrm{y} 3)$ respectively denote the three vanishing points. The orthocentre of the three vertices be denoted by $\mathrm{Cv}(\mathrm{xc}, \mathrm{yc})$. From figure 11, the lines joining the three vertices of the vanishing point, constitute the three horizon lines. This also is the center of focus of the image. Thus from the images acquired from unknown camera parameters, it is possible to find the center of focus of the camera from its image which contain 3 vanishing point, from equations 23 and 24. Thus roll rectification from such images is brought about with the following procedure.

The image for camera roll correction is subjected to vanishing point determination. From the vanishing points obtained, the orthocenter of the triangle that could possibly be formed by joining the three vertices is found out by equations 23 and 24. By doing this, the camera is partially calibrated for the center of focus of the camera. Thereafter, the camera center is made as the image center by adding the padding strips of zero values to the corner of the image. Thus the camera roll is rectified from the image by rotating the image in reverse direction of the roll by an angle equal to the slope of the horizon lines formed by the horizon line with minimum slope. The algorithm for the same is given as below.

Some of the results for images with the unknown camera parameters for roll rectification are shown in table 2 . 


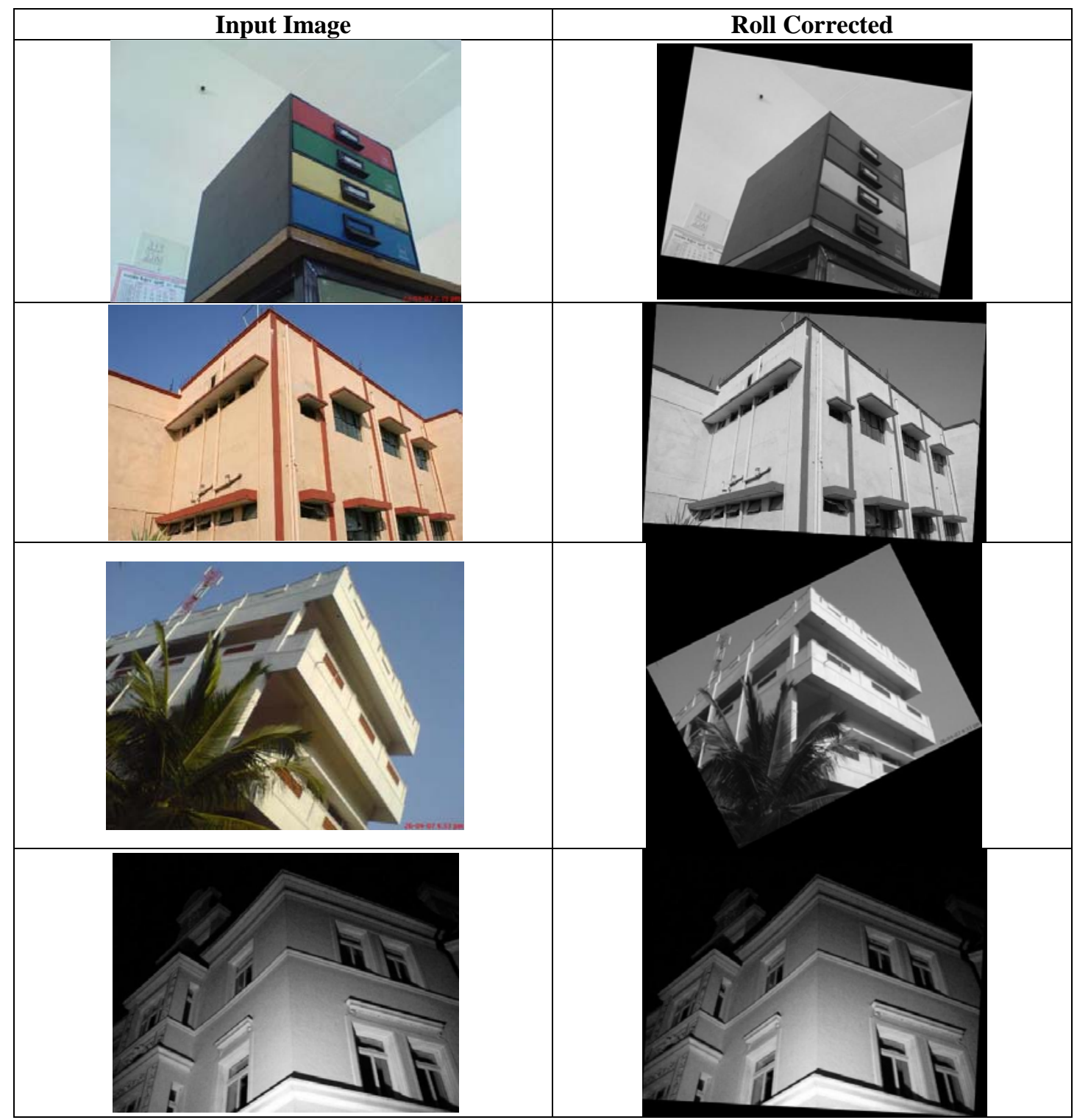

Table 2: The first column contains the original images. The camera roll corrected images are filled up in the second column

\section{Algorithm:}

Step 1. Read the initial image.

Step 2. Determine the vanishing points.

Step 3. Find the orthocenter of the triangle formed from the three vanishing points.

Step 4. Add padding zeros to the image by the sides of the image in order to make the center of focus as the image center.

Step 5. Compute the slope of the base horizon line which is having least slope (case of 3 horizon lines due to the presence of 3 vanishing points) and compute the angle of inclination to the horizontal axis of the image.

Step 6. Rotate back the image by an angle computed in the previous step. 


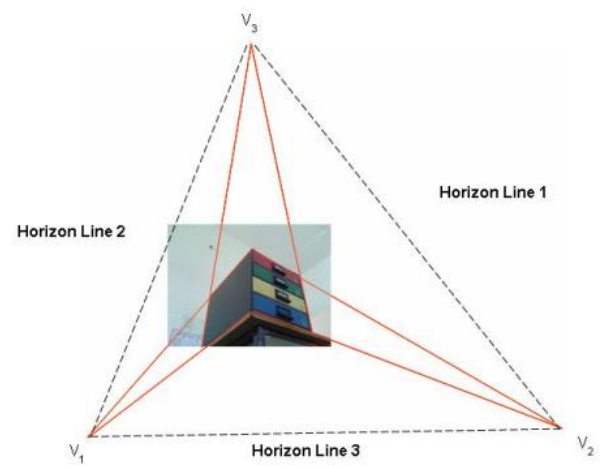

Figure 11: Depiction of 3 vanishing points and the corresponding horizon lines formed.

The entire work stands pivoted on the principle of vanishing point detection. The vanishing points are supposedly are to be considered for the correction of camera roll in all cases. In natural scenes except for the building structures and structures with parallel and vertical edges in the image we find the existence of vanishing point prominently. But in cases of images having fewer or no straight lines and edges it is a challenging task to find the vanishing points itself. Hence this roll correction stands as the challenging task in cases of the images having fewer or no straight and parallel edges in them.

\section{Computational Complexity of the Entire Algorithm:}

The method we employ for the camera roll correction involves the following steps, viz.

1. Vanishing point determination.

a. Using the center of focus of the camera parameters of images having two vanishing points.

b. Finding the orthocenter of the triangle formed in case of 3 vanishing point images.

2. Determination of slope of the horizon line (the horizon having minimum slope in case of image having 3 vanishing points).

3. Making camera center as image center.

4. Image rotation.

The computational complexity of the entire algorithm is the summation of the individual computational complexities of the above mentioned steps. Steps of finding the orthocenter, determination of slope of the horizon line and bringing the camera center as image center in this roll rectification algorithm take constant time for variable inputs. They include equations for direct substitutions to solve them, taking constant time period.

Although there are many methods reported in the literature for image rotation, which are capable of performing fast image rotation, we adhere to the simplest form of image rotation for demonstration purpose. The time taken for the execution of the image rotation algorithm is proportional to the image size as all the pixels are multiplied by the rotation matrix. Hence the complexity of the image rotation for an image of size $(m \times n)$ is $\mathcal{O}(m \times n)$.

The other step which influences the change in computational complexity is as follows.

\subsection{Computational complexity of the vanishing point determination procedure adopted in this work:}

For ' $n$ ' number of line segments, the pseudo code of the algorithm of the vanishing point determination is shown in table 3. The calculations of vanishing points are only for ${ }^{n} C_{2}$ calculations, and hence the loops structures for the calculations are as follows. 
for $i=1$ to $(n-1)$

$$
\begin{aligned}
& \text { for } \mathrm{j}=(\mathrm{i}+1) \text { to } \mathrm{n} \\
& \qquad \begin{array}{c}
\mathrm{X}=\frac{\rho_{1}-\mathrm{Y} \cdot \sin \theta_{1}}{\cos \theta_{1}}=\frac{\rho_{2}-\mathrm{Y} \cdot \sin \theta_{2}}{\cos \theta_{2}} \\
Y=\frac{\left(\left(\frac{\rho_{1}}{\cos \theta_{1}}\right)-\left(\frac{\rho_{2}}{\cos \theta_{2}}\right)\right)}{\tan \theta_{1}-\tan \theta_{2}}
\end{array}
\end{aligned}
$$

next j

next $\mathrm{i}$

Table 3: Pseudo code of the algorithm for finding the vanishing point.

The total time of computation on the worst case is thus a lesser than half of $\mathrm{n}^{2}$ Values.

Thus the total time complexity of the algorithm is of the order $\mathcal{O}(n *(n-1)) / 2$ or $\mathcal{O}\left(\frac{n^{2}}{2}-\frac{n}{2}\right)$.

Ignoring the lower degree terms, the worst time complexity of the algorithm is at the most $\mathcal{O}\left(\frac{\mathrm{n}^{2}}{2}\right)$.

\section{Discussions}

\subsection{Computation of the Fabrication Error:}

For the image in figure 7(a) (acquired using Sony Ericsson K750i), upon calibrating the camera using [13] [20], the center of focus of the camera is found out to be present at $(337,227)$ for the image of size $640 \times 480$. But for the image sensor used, maximum size of the image from the center can be only 320x240.

Thus the maximum possible radial drift that can be caused to make the center completely away from the image center is given by ' $d$ ', and that is,

$$
M_{d}=\sqrt{320^{2}+240^{2}}=400
$$

Drift from the optical center of the lens to the image center is given by the distance formula and equated to $O_{d}$,

$$
O_{d}=\sqrt{(337-320)^{2}+(227-240)^{2}}=17.263
$$

Therefore, the percentage inaccuracy or the fabrication error wrt the maximum drift of the optical center to the image sensor is given by equation 25 .

$$
\text { Fabrication error }=\left[1-\frac{M_{d}-O_{d}}{M_{d}}\right] \times 100
$$

Thus,

$$
\text { Percentage inaccuracy or Fabrication error }=4.316 \%
$$

\subsection{Computation Time with Increased Number of Line Segments:}

The lines used for the intersection of points are calculated in Polar Co-ordinate system. Images acquired from the cameras having a particular roll can have any angle and hence the vanishing point determination technique we have presented is robust enough to handle all possible slopes in the straight line segments in the image.

Furthermore, from the calculation of the complexity of the camera roll correction algorithm, it can be said that the camera roll correction depends mainly on the number of straight line segments present in the image contributing for the vanishing points. If the number of straight line segments increase, the computation time taken has a quadratic 
growth. The calculations of vanishing points are only for ${ }^{n} C_{2}$ calculations and hence the total time of computation on the worst case is thus lesser than half of $n^{2}$ values, where ' $n$ ' is the number of straight lines present. Furthermore, upon determination of the vanishing points, the algorithm takes constant computation time based on the number of pixels present in the image to be handled. This has been presented in section 6 .

\subsection{Application of Camera Roll Correction:}

By using multiple-image technique, panoramic images can be produced with conventional cameras using image mosaicing principles to make multi-image panoramic photography readily available to the photography enthusiasts. Many of the modern day cameras (eg. SE K750i, Samsung Omnia 1900 etc.) are equipped with the panoramic view generation feature in them. Usually these devices are hand held devices and are subjected to camera roll, tilt and pan during image acquisition. In these cameras, they need to be panned horizontally with zero roll in camera to generate the panoramic view. This leads to generation of panoramic view in a horizontal direction. While acquiring the images, there are chances of camera roll happening as the devices are hand held and no proper fixture is used. Hence the image needs to be positioned accurately each and every time the split images are acquired. The use of camera roll correction system can solve this issue by automatically aligning all split images horizontally during the image acquisition stage. This can also help in quicker photography in panoramic view generation.

\section{Conclusion}

In the current paper we are focusing mainly on the correction of camera roll in a perspectively distorted image to bring the camera rolled images containing tilted objects to a necessary vertical position. Here we employ the rectification of the horizon line formed from the vanishing points in order to bring back the image rotation caused from the camera roll. We proposed a novel idea of auto roll rectification algorithm for a camera system based on the vanishing points. This can be readily used in camera's software to build an intelligent auto roll rectifying feature in it. The work finds future expansion in cases of images having lesser straight edges or no straight edges of the lines contributing for vanishing points.

\section{References}

[1] Max Born, Emil Wolf, Principles of Optics, 7th Edition, Cambridge Univ. Press, 1999.

[2] "Photographic Lenses", Photographic, April 1991, pg:56-57, 1999.

[3] Collins Douglas, The Story of Kodak, Harry N. Abrams Inc. Publishers, 1990.

[4] A. Bors, I. Pitas, W. Puech, J. Chassery, "Perspective Distortion Analysis for Mosaicing Images Painted on Cylindrical Surfaces", IEEE Proc. of International Conference on Acoustics, Speech \& Signal Processing (ICASSP'97), Volume 4.

[5] C. Grimm, K. Singh, N. Sudarsanan, “The iBar: A Perspective-Based Camera Widget”, Technical Report WuCSE2004-32, Wash. Univ. in St. Louis, 2004.

[6] L.S.Harris, Naval Ordnance and Gunnery, Volume 2, Fire Control, Chapter 16 Radar \& Optics, 1992.

[7] C. Preusche, J. Hoogen, D. Reintsema, G. Schmidt, "Flexible Multimodal Telepresent Assembly Using a Generic Interconnection Framework", IEEE Proc. of International Conference on Robotics and Automation 2002 (ICRA2002), Volume 4. Issue 2002, Pg: 3712-3718.

[8] E. H. Elsayed, T. Ahmed Moumen, Aly A. Farag, The CardEye: A Trinocular Active Vision System, Springer Berlin / Heidelberg, Vol 2095/2001. 
[9] David P. Stern, Distance to the Horizon, Article for general reading available at the NASA web link wwwistp.gsfc.nasa.gov/stargaze/shorizon.htm

[10] M. J. Magee, J. K. Aggarwal, “Determining Vanishign Points from Perspective Images”, International Journal of Computer Vision Graphics and Image Processing, Vol 2, Pp: 256-267, May 1984.

[11] S. T. Barnard, "Interpreting Perspective Images", International Journal on Artificial Intelligence, No. 4, Pp: 435-462, Nov. 1983.

[12] E. Lutton, H. Maitre, J. Lopez-Krahe, "Contributions to the Determination of Vanishing Points Using Hough Transform", IEEE Transactions on Pattern Analysis and Machine Intelligence, Vol. 16, Issue 4, Pg:430-438, Apr. 1994.

[13] Avinash N., Murali S., "Camera Center Estimation Using Vanishing Points", IEEE 1st International Conference on Signal and Image Processing, 2006., Volume: 1, On page(s): 467, 2006.

[14] J. S. Dobrovolny, D. C. O’Bryant, Graphics for Engineers, 2nd Edition, John Wiley \& Sons Inc. Canada, 1984. ISBN 0-471-87124-9.

[15] Janne Heikkila, Olli Silven, “A Four Step Camera Calibration Procedure with Implicit Image Correction”, CVPR ‘97, 1997.

[16] Z. Zhang, "Flexible Camera Calibration by Viewing a Plane from Unknown Orientation”, ICCV '99, 1999.

[17] A. Ruiz, P. E. Lopez-de-Teruel, G. Garcia-Mateus, "A Note on Principal Point Estimability", 16th International Conference on Pattern Recognition (ICPR '02), Vol. 2, Pg:20304, 2002.

[18] Criminisi, A. Reid, I. Zisserman, A., "Single View Metrology", The Proceedings of the Seventh IEEE International Conference on Computer Vision, 1999., Volume: 1, On page(s): 434-441, 1999.

[19] Douglas, A. Kerr, P.E., "The Proper Pivot Point for Panoramic Photography”, The Pumpkin, Issue: 2, Dec. 6, 2005.

[20] Avinash N., Murali S., "Perspective Geometry Based Single Image Camera Calibration", Journal of Mathematical Imaging and Vision, Volume 30 , Issue 3 (March 2008) Pages: 221 - 230

[21] Avinash N., Nagendra, Murali S., "Multiple Vanishing Point Determination”, in proc. "IEEE International Conference on Advances in Computer Vision and Information Technology”, Aurangabad, India, Nov 2007.

[22] A. Zisserman, R. Hartley, "Multiple View Geometry in Computer Vision”, Cambridge University Press. ISBN: 0521540518 .

[23] Canny, J., A Computational Approach To Edge Detection, IEEE Trans. Pattern Analysis and Machine Intelligence, 8:679-714, 1986.

[24] Henry E. Garret, "Statistics in Psychology and Education”, Chapter 2: Measures of Central Tendency. Vakils, Feffer \& Simons Pvt. Ltd. 1973. pp. 27-41.

[25] Richard O. Duda, Peter E. Hart, David G. Stork, "Pattern Classification, Second Edition", Wiley Text Books publishers, ISBN: 9814126020. 